significantly $(p<0.0001)$ higher in DM/PM $(n=361,17.3 \%)$ with respect to controls $(\mathrm{n}=1,018,10.0 \%)$.

Concerning prognosis, ANA positivity in PM/DM was associated with a better prognosis for all cancers (OR 0.39 [95\% 0.24-0.63], $\mathrm{p}=0.0001$ ). For individual cancer types; thyroid cancer (OR 0.39 [95\% 0.24-0.63], $\mathrm{p}=0.0001$ ), gastric cancer (OR 0.40 [95\% 0.25-0.64], $\mathrm{p}=0.0001$ ), kidney cancer (OR 0.39 [95\% 0.24-0. 62], $p=0.0001$ ), acute leukaemia (OR 0.40 [95\% 0.25-0.65], $p=0.0002$ ), non-Hodgkin's lymphoma (OR 0.39 [95\% 0.25-0.63], $p=0.0001$ ), but not for myelodysplastic syndrome.

The main cancers linked to PM/DM were thyroid cancer (OR $3.17[95 \% \mathrm{Cl} 2.27$ 4.43]), gastric cancer (OR 5.96 [95\% Cl 4.24-8.38]), kidney cancer (OR 3.83 [95\% $\mathrm{Cl} 1.02-14.31], \mathrm{p}=0.0462$ ), and myelodysplastic syndrome (OR 2.01 [95\%Cl 1.17-3.46], $p=0.0111$ ). Regarding gastric cancer, positivity for anti-RNP (OR 5.68 [95\% Cl 3.02 to 10.71 ], $\mathrm{p}<0.0001$ ), anti-SSA (OR 21.99 [95\% $\mathrm{Cl} 11.21$ to 43.14], $\mathrm{p}<0.0001$ ), and anti-Jo1 (OR 12.23 [95\%Cl 7.12 to 21.01], $\mathrm{p}<0.0001$ ) was associated with a higher risk of cancer development.

Conclusion: ANA positivity is an independent predictor of favorable prognosis in PM/DM patients with cancer, possibly suggesting that cancer directed humoral autoimmunity may have some benefit. Therefore, humoral autoimmunity in SSc and $\mathrm{PM} / \mathrm{DM}$ is a broad mechanism that confers a survival advantage and is relevant for disease understanding and elucidating optimal anti tumoural immunity in the current age of cancer immunotherapy.

References:

[1] Watad A, McGonagle D, Bragazzi NL, Tiosano S, Comaneshter D, Shoenfeld $\mathrm{Y}$, Cohen $\mathrm{AD}$, Amital $\mathrm{H}$. Autoantibody status in systemic sclerosis patients defines both cancer risk and survival with ANA negativity in cases with concomitant cancer having a worse survival. Oncoimmunology. 2019 Mar 24;8(6):e1588084

Disclosure of Interests: Abdulla Watad: None declared, Dennis McGonagle Grant/research support from: Janssen Research \& Development, LLC, Merav Lidar: None declared, Nicola Luigi Bragazzi: None declared, Doron Comanesther: None declared, Arnon Cohen: None declared, Howard Amital: None declared

DOI: 10.1136/annrheumdis-2020-eular.5977

\section{OP0254 CHROMATIN CONFORMATION SIGNATURE ANALYSIS IN EARLY VS LATE SCLERODERMA PHENOTYPES}

M. Galloway ${ }^{1}$, E. Hunter ${ }^{2}$, A. Akoulitchev ${ }^{2}$, S. Vigneswaran ${ }^{1}$, B. Abdi ${ }^{1}$, C. Denton ${ }^{1}$, D. Abraham ${ }^{1}$, R. Stratton ${ }^{1} .{ }^{1}$ UCL Division of Medicine Royal Free Campus, Centre for Rheumatology and Connective Tissue Diseases, London, United Kingdom; ${ }^{2}$ Oxford Biodynamics Plc, Oxford, United Kingdom

Background: Systemic sclerosis (scleroderma, SSc) is a heterogeneous dis ease in which clinical outcomes vary widely. Predicting outcomes on an individual basis remains challenging despite progress made through autoantibody analysis and gene expression profiling. Effective targeted therapies are evolving and accurately predicting outcomes is important to enable patient stratification for therapy.

Chromatin Conformation Signature (CCS) profiling of peripheral blood for systemic epigenetic deregulations could be used for such a purpose. The EpiSwitch platform offering high throughput and resolution chromosome conformation (3C) capture detects significant regulatory changes in 3D genome architecture and maps long range interaction between distant genomic locations. This then reveals the spatial disposition and physical properties of the chromosome, such as chromatin loops and inter-chromosomal connections, which have a role in network organization and genetic epistasis controlling gene expression. EpiSwitch automated platform has been successfully utilised in patient stratification in RA, MS and other indications.

This methodology could be applied to patients with SSc to identify CCS associated with different phenotypes and may ultimately be used to stratify and identify patients into pathogenic subtypes.

Objectives: We aimed to determine significant CCSs associated with early and late phenotypes of SSc.

Methods: The EpiSwitch-based chromosome conformation capture (3C) method was applied to blood samples from early phenotype, and late phenotype SSc patients. Intact nuclei were isolated from peripheral blood mononuclear cells and subjected to formaldehyde fixation resulting in crosslinking between physically touching segments of the genome via contacts between their DNA bound proteins. For quantification of cross-linking frequencies, the cross linked DNA was digested and then subjected to ligation. Cross-linking was then reversed and individual ligation products detected and quantified by EpiSwitch custom oligo array annotated across the whole genome to the anchoring sites of 3D genome architecture.

Results: 7 significant CCSs were found over the HLA-C, HLA-B and TNF regions on Chromosome 6 in the early phenotype. The top 8 pathways for genetic locations associated to the CCSs are shown in Table 1.
Table 1. Top 8 pathways for genetic locations associated to significant CCS for the early phenotype.

GeneSe

Natural Killer cell mediated cytotoxicity

Immunoregulatory interations between a lymphoid cell and a non-lymphoid cell

Antigen Processing \& presentation

Phagosome

Graft versus host disease

Type 1 diabetes mellitus

Osteoclast differentiation

Class $1 \mathrm{MHC}$ mediated antigen processing \& presentation

2 significant CCSs were found centred around the IFNG region of chromosome 12 in the late phenotype. The top 8 pathways for genetic locations associated to significant CCSs are shown in Table 2.

Table 2. Top 8 pathways for genetic locations associated to significant CCS for the late phenotype.

GeneSet

Surfactant metabolism

IL12 signalling mediated by STAT4

Protein digestion \& absorption

Calcineruin regulated NFAT dependent transcription in lymphocytes

Transcriptional misregulation in cance

Kaposi's sarcoma associated herpes virus infection

IL2 mediated signalling events

Inflammatory bowel disease

Conclusion: Significant CCSs, as part of 3D genomic regulatory control, and their associated pathways for the genetic locations, were identified in both late and early phenotypes. There were distinct CCSs in the early phenotype compared to the late suggesting the CCSs change as the disease progresses and varies between phenotypes. If CCSs could be linked to each clinically defined subgroup across a SSc cohort they could be used as a biomarker tool to predict outcome and progression in patients.

Disclosure of Interests: Megan Galloway: None declared, Ewan Hunter: None declared, Alexandre Akoulitchev: None declared, Shivanee Vigneswaran: None declared, Bahja Abdi: None declared, Christopher Denton Grant/ research support from: GlaxoSmithKline, Inventiva, CSF Behring, Consultant of Roche-Genentech, Actelion, GlaxoSmithKline, Sanofi Aventis, Inventiva, CSL Behring, Boehringer Ingelheim, Bayer, David Abraham: None declared, Richard Stratton: None declared DOI: 10.1136/annrheumdis-2020-eular.6323

\section{PARE abstract session}

\section{OP0255-PARE USING AN EDUCATIONAL APPLICATION TO FACILITATE UNDERSTANDING OF THE ANATOMY AND FUNCTION OF THE BRAIN AND TO EXPLORE THE EFFECTS OF CLINICAL FATIGUE FROM A PATIENT PERSPECTIVE}

J. Zurowski ${ }^{1}$, C. Wright ${ }^{2}$, N. Basu ${ }^{2}$, M. Poyade ${ }^{1}$, L. Bennett ${ }^{2}{ }^{1}$ The Glasgow School of Art, School of Simulations and Visualisation, Glasgow, United Kingdom; ${ }^{2}$ University of Glasgow, Institute of Infection Immunity and Inflammation, Glasgow, United Kingdom

Background: Rheumatic and musculoskeletal diseases are a group of devastating autoimmune disorders that all commonly share the debilitating symptom of fatigue. Despite the fact that fatigue can often cause some of the greatest impairments to quality of life, it is frequently reported by patients as the least successfully managed symptom of these conditions.

Fatigue is routinely misunderstood within the general population, with many people using the word fatigue as a synonym for tired. Fatigue is not the same as tiredness, which is a normal state that is experienced by most of the population, therefore it is important to help the general public understand what fatigue actually is and how it imposes consequences and limitations on those who suffer from it. To aid this understanding an educational application has been created to reinforce the patient perspective of living with fatigue. Furthermore, this application will also aid the understanding of brain anatomy and function, using Augmented Reality (AR), as research has now shown that brain function may be altered in the state of fatigue. Currently, educational AR applications show great potential for increasing comprehension and understanding of complex concepts. AR expands use engagement by enhancing the learner's enjoyment and enriching their learning environment. We hope to utilise this technology in the education of fatigue. 